\title{
Endometriosis; Contribución al Estudio de su Incidencia en Nuestro Medio
}

\author{
Doctor Hernando Amaya-León \\ Profesor Agregado de la Facultad Nacional de Medicina, Bogotá, Colombia. \\ Del Departamento de Ginecologia y Obstetricia del "Consultorio de Especialistas". \\ Carrera 13, No 43-23. - Bogotá, Colombia.
}

Desde hace años he tenido la impresión de que la endometriosis constituve un hallazgo raro en nuestras mujeres. En algunas ocasiones pensaba en incomxetencia de los cirujanos en reconocerla durante el acto operatorio y en otras ima ginaba que los histologos eran incapaces de diagnosticarla. Durante mi viaje el año pasado a Norte América, en el que recorrí centros hospitalarios de diversos sectores de esa zona, tuve la oportunidad de observar que tal entidad aunque en general mucho más frecuente en ese país que en el nuestro, constituía un problema de incidencia muy diverso en los diferentes lugares visitados, aumentando el número de casos en forma apreciable a medida que iba llegando al norte de los Estados Unidos. Esto me hizo pensar que, en nuestro medio si bien podían existir fallas en algunos cirujanos e histólogos, la evidencia tenía que ser hallada en la poca frecuencia de la endometriosis debida a factores desconocidos, quizás de origen racial o ambiental.

Revisando bibliografias se encuentran datos informativos curiosos:

Sampson (1-2), la encuentra en 104 de 466 pacientes operadas, o sea $22 \%$. Meigs ( 3 ). en $19+1$ afirma su hallazgo en el $28 \%$ de 400 pacientes privadas sometidas a laparotomía y tan sólo en el $5 \%$ de las operadas en su servicio del Massachussets General Hospital; estos datos los confirma años mas tarde con muy ligeras variaciones. En cambio $\mathrm{Te}$ Linde $\left({ }^{4}\right)$, de Baltimore, halla menos de ese $5 \%$ en sus servicios del Johns Kopkins Hospital; y recuérdese de paso, que la incidencia de negros en esta ciudad es mucho mayor que en Boston; ésto tendria similitud con las estadísticas de Blinik y Meredino $\left({ }^{5}\right)$, del Harlem Hospital de New York, de pacientes casi exclusivamente de raza negra quien habla 
tan sólo del $0.1 \%$ de casos de endometroisis al revisar todas las mujeres someti das a cuidados ginecológicos en dicho hospital hasta 1950. Nowak (6) también de Baltimore, considera frecuente la entidad, pero no en la forma tan alta como la sugirió inicialmente Sampson. Selye $\left({ }^{7}\right)$, del Canadá, estima que alrededor del $20 \%$ de todas las mujeres que requieren operación abdominal por cualquier causa, sufren de endometriosis; y Fallas ( $(8)$, de Los Angeles, California, reporta 150 casos de pacientes privadas entre 388 operadas por diferentes condiciones ginecológicas, o sea el $38 \%$, incidencia ésta la más alta de las revisadas por mí. Hollmes ( ${ }^{9}$ ), de Atlanta, Georgia, es uno de los pocos autores del sur de los Estados Unidos que refiere una notable frecuencia de endometriosis en sus pacientes: el 26\% de todas las operaciones ginecológicas. En cambio, Kelly y Schlademan $\left({ }^{11}\right)$, tan sólo comprueban 179 estudios histológicos positivos en 1.991 ope raciones practicadas, o sea $9 \%$.

No me fue posible hallar gran cosa en bibliografía de otros países, pero debo traer a cuento las cifras de Medrazo Bassani (11), en Méjico, quien no puede hallar sino 30 casos en 1.483 laparotomías, o sea menos del $3 \%$, y las de Brzezinki y Bromberg ( ${ }^{12}$ ), de Israel, quienes en forma similar no encuentran sino 42 casos comprabados histológicamente de 1.027 operaciones ginecológicas, o sea $4.1 \%$.

Si bien, como lo afirma Wharton $\left({ }^{13}\right)$, la endometriosis se diagnostica más frecuentemente en la sala de cirugía que en el laboratorio de Patología, estas cifras halladas distan mucho de las mencionadas por los primeros autores què describieron la entidad.

Yo presento en este trabajo, una revisión de historias de mi archivo privado. Sobre 1.631 pacientes particulares vistas de 1945 a 1953, fueron sometidas a intervención abdominal por condición ginecológica 171 enfermas, entre las cuales fueron practicados 110 examentes histológicos.

Se encontraron 10 casos de endometriosis cumprobados, o sea:

$0.6 \%$ del total de enfermas vistas, y el $5.8 \%$ de los casos intervenidos; en los 60 casos sin estudio histológico, no había ninguno en dende se sugiriera la endometriosis.

Hubo 16 casos entre los 1.631 enfermas en donde se sospechó clinicamente la enfermedad, pero en las que no hubo indicación operatoria o ésta fue rechazada.

Como localización de esas lesiones endometriales, tenemos:

4 en ovarios. $\mathrm{t}$

3 en útero (adenomiosis).

1 en ovario y Douglas.

2 en tubos. 
De las 10 enfermas:

5 eran multíparas.

2 vírgenes.

3 casadas e infértiles.

De las infértiles:

1 fue operada a los 47 años por otra condición ginecológica.

1 fue operada por salpingitis crónica bilateral, que creaba situación de esterilidad irreversible; además se encontró endometriosis ovárica.

1 fue operada por oclusión tubárica, y en la trompa derecha se encontró endometriosis; resección parcial y salpingoplastia; actualmente en observa. ción y con permeabilidad bilateral neta de las trompas.

De las cifras encontradas en este trabajo al ser comparadas con las anteriormente citadas, cabe la sugerencia de pensar que la endometriosis parece más frecuente en los grupos de mujeres de raza con neto predominio blanco; no hay duda que factores de otro orden deben influír notoriamente, y podría pensarse así que la contracepción prolongada debe ser estudiada con especial cuidado a este respecto.

Comprendo que los datos presentados en este trabajo son insuficientes para aclarar el problema de la incidencia de la endometriosis en nuestro medio; por ello, invito a los ginecólogos del país para que revisando sus estadísticas privadas y hospitalarias, podamos hallar cifras de mayor exactitud.

\section{Resumen:}

19-Revisando 1.631 historias clínicas de enfermas privadas (1945-1953) se encuentran 10 casos de endometriosis con comprobación histológica, sobre un total de 171 operaciones abdominales.

2. - Esto constituye el $0.6 \%$ del total de enfermas y el $5.8 \%$ de las sometidas a laparotomía.

$3^{\circ}$ - Se piensa en la infuencia racial en la etiología de la endometriosis, lo mismo que en el uso prolongado de métodos contraceptivos.

40-Las cifras del autor necesitan la comprobación de estadísticas privadas y hospitalarias de otros ginecólogos del país. 


\section{B I B L I O G R A I A :}

1.-Sampson, J. A.: "The Life of Ovarian Haematomas of Endometrial Type" Am. J. Obst. Gynec., 4: 451, Nov. 1922.

2.-Sampson, J. A.: "Perforating Haemorrhagic Cysts of the Ovary". - Arch. Surg., 3: 245. - 1921.

3.-Meigs, J. C.: "Endometriosis; its significance". - Ann. Surf., 114: 866. - 1941.

4.-Te Linde, R. W.: "Operative Gynecology". - J. B. Lippincott Co., - 1946.

5. -Blinick and Meredino: (Cit. Year Book of Obst. Gynec, J. P. Greenhill, p. 435, 1952). Am. J. Surg., 81: 635. - 1951.

6. -Novak E.: "Test Book of Gynecology". - Williams and Wilkins Co., Baltimore, 1948.

7.-Selye H.: "Textbook of Endocrinology". - Acta Endoerinológica, Montreal. Canadá, 1947.

8.-Fallas, Roy E.: "Endometriosis: Study of 150 Privates cases". - West. J. Surg., 59: 448. - 1951 .

9.-Hollmes, W. R.: “Endometriosis”. - Am. J. Obst. Gynec., 43: 255. - 1942.

10.- Kefly, F. J.; Schlademan, K. R.: "Endometriosis; its Surgical significance". Surg. Gynec. Obst., 88: 230. - 1949.

11. -Madrazo, Bassani M.: "Endometriosis". - Ginec. y Obst., Méjico, 3: 13. - 1948

12. - Brzezinki y Bromberg.: (Cit. Year Book of Obst. Gynec., J. P. Greenhill, p. 435, 1952). Acta Med. Orient., 10: 173. - 1951.

13. -Wharton, L. F.: "Gynecology with a section on Female Urology". - W. B. Saunders Co., Philadelphia, 1943. 